\title{
Reversible Watermarking Method for JPEG Images
}

\author{
Akira SHIOZAKI $^{\dagger a)}$, Motoi IWATA ${ }^{\dagger}$, and Akio OGIHARA ${ }^{\dagger}$, Members
}

SUMMARY In this letter, we propose a simple reversible watermarking method for JPEG images using the characteristics of JPEG compression. The method embeds a watermark into a JPEG image, and it extracts the watermark from the watermarked JPEG image and at the same time can recover the watermarked JPEG image to an original unwatermarked JPEG image. Moreover we investigate the number of embeddable blocks, the quality of watermarked images, and the increase of file-size by embedding a watermark.

key words: watermark, reversible watermarking, JPEG image

\section{Introduction}

Digital watermarking schemes are techniques that embed additional data into digital contents so that the distortion by embedding them is perceptually undetectable [1]. Usually, additional data are embedded within permissible limits of distortion. However, there are such cases where no distortion is allowed. So the techniques called reversible watermarking have been proposed [2]-[5]. Reversible watermarking schemes embed additional data into an image, and can reconstruct the original image from the watermarked image as well as extract the embedded data.

Reversible watermarking methods have been presented now for raw images such as bitmap-formatted (BMP) images. This is because non-reversibly compressed images such as JPEG images have already been degraded by compression and so the reconstruction seems to be meaningless.

However, in some uses, it seems that there are cases where secret information is embedded into a JPEG image and where no distortion of the cover image is desirable. In those cases, reversible watermarking schemes for JPEG images are useful. For example, medical images are often filed in JPEG format and so it is desirable that the original JPEG images are reconstructed from the watermarked JPEG images in which private information is embedded for privacy protection. Sakai, et al. have applied the reversible watermarking method [3] to JPEG images [6], and Xuan, et al. have proposed a reversible data hiding scheme for JPEG images [7].

This letter proposes a new simple reversible watermarking method for JPEG images that not only extracts embedded data from the watermarked JPEG images but also reconstructs the original JPEG images from the watermarked

Manuscript received December 12, 2007.

Manuscript revised February 27, 2008.

${ }^{\dagger}$ The authors are with the Graduate School of Engineering, Osaka Prefecture University, Sakai-shi, 599-8531 Japan.

a)E-mail: shiozaki@cs.osakafu-u.ac.jp

DOI: 10.1093/ietisy/e91-d.7.2068 images. The proposed method uses the characteristics of JPEG compression.

\section{Watermark Embedding}

A JPEG image is decoded with Huffman coding and runlength coding to get quantized DCT coefficients. A watermark is embedded in the quantized DCT coefficients and the quantized DCT coefficients are encoded with run-length coding and Huffman coding again to get a watermarked JPEG image. A watermark can be embedded into luminance component $(\mathrm{Y})$ and color difference components $(\mathrm{Cb}, \mathrm{Cr})$. We describe the method in the case of embedding a watermark in one of the three components.

Let $w_{j} \in W=\{0,1,2, \cdots, 2 n-1\}(j=1,2, \cdots, N)$ be the symbols of a watermark to be embedded, where $n$ and $N$ are positive integer. When $n=2^{k-1}, k$ bits can be embedded in an $8 \times 8$-pixel block. The number of embeddable blocks is different in each image.

Figure 1 shows the principle of watermark embedding, and Fig. 2 shows the schematic view of watermark embedding. A watermark is embedded as follows:

Step 1) Let $j=1$ and select a block in which $w_{j}$ is going to be embedded.

Step 2) Let $C_{i}(i=0,1, \cdots, 63)$ be quantized DCT coefficients of the $j$-th $8 \times 8$-pixel block in zigzag-scanning order. Search $m$ such that $C_{m} \neq 0$ and $C_{i}=0(i=$ $m+1, \cdots, 63)$.

Step 3) When $m<63$,

$$
\begin{array}{lll}
C_{m+1} \leftarrow w_{j}-n+1 & & \text { for } w_{j} \geq n \\
C_{m+1} \leftarrow w_{j}-n & & \text { for } w_{j}<n .
\end{array}
$$

When $m=63$, a watermark does not embedded and

$$
\begin{array}{ll}
C_{63} \leftarrow C_{63}+n & \text { for } C_{63}>0 \\
C_{63} \leftarrow C_{63}-n & \text { for } C_{63}<0 .
\end{array}
$$

Then $j \leftarrow j+1$.

Step 4) Select the next block, and repeat from Step 2 to Step 3 until $w_{N}$ is embedded.

\section{Watermark Extraction and Recovery of JPEG Image}

The JPEG image in which a watermark has been embedded is decoded with Huffman coding and run-length coding, and an embedded watermark is extracted from the quantized 


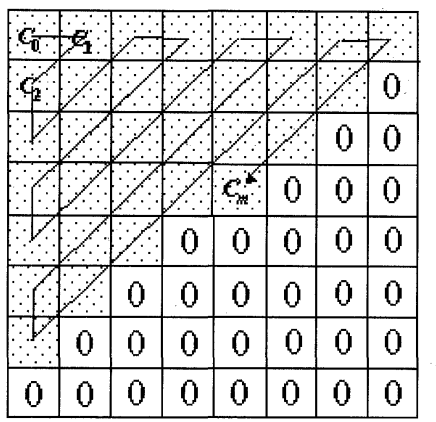

(a) Before embedding

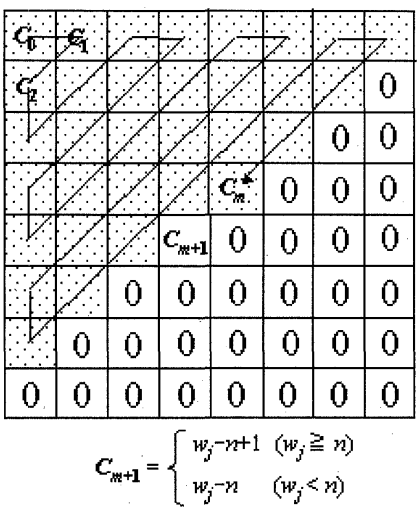

(b) After embedding

Fig. 1 Principle of embedding watermark (modification of DCT coefficients)

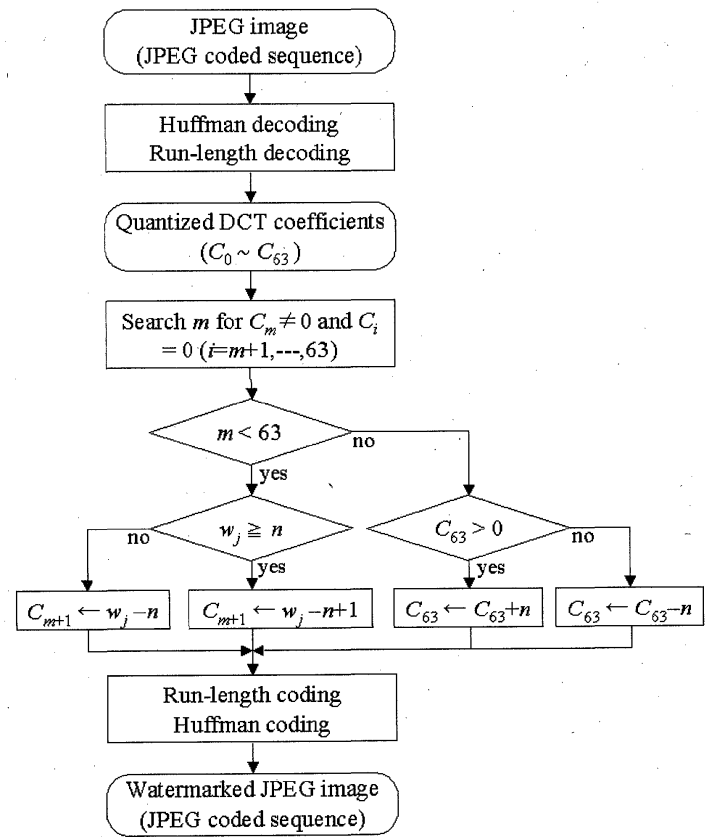

Fig. 2 Schematic view of embedding watermark.

DCT coefficients of $8 \times 8$-pixel blocks as described below. Then the quantized DCT coefficients are encoded with runlength coding and Huffman coding again to get the original JPEG image. Figure 3 shows the schematic view of extract-

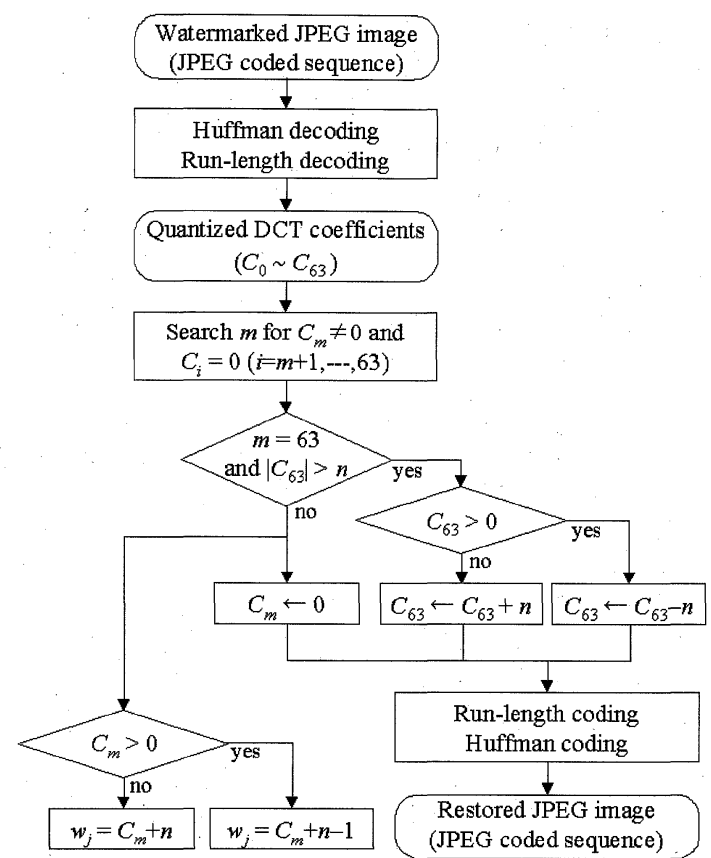

Fig. 3 Schematic view of extracting watermark and recovering JPEG image.

ing watermark and recovering JPEG image.

Step 1) Let $j=1$, and select the block in which $w_{j}$ has been embedded.

Step 2) Let $C_{i}(i=0,1, \cdots, 63)$ be quantized DCT coefficients of the $j$-th $8 \times 8$-pixel block in zigzag-scanning order. Search $C_{m}$ such that $C_{m} \neq 0$ and $C_{i}=0(i=$ $m+1, \cdots, 63)$.

Step 3) When $m=63$ and $\left|C_{63}\right|>n$,

$$
\begin{array}{ll}
C_{63} \leftarrow C_{63}-n & \text { for } C_{63}>0 \\
C_{63} \leftarrow C_{63}+n & \text { for } C_{63}<0 .
\end{array}
$$

Otherwise,

$$
\begin{array}{ll}
w_{j}=C_{m}+n-1 & \text { for } C_{m}>0 \\
w_{j}=C_{m}+n & \text { for } C_{m}<0,
\end{array}
$$

and $C_{m} \leftarrow 0$.

Then $j \leftarrow j+1$.

Step 4) Select the next block and repeat from Step 2 to Step 3 until $w_{N}$ is extracted.

\section{Performance}

We investigated the performance of the proposed method for several JPEG qualities. In the experiment, we used bitmap (BMP) color images with $256 \times 256$ pixels and 256 brightness levels for $\mathrm{R}, \mathrm{G}$ and $\mathrm{B}$ components respectively, which are shown in Fig. 4. A watermark was made from a uniform random number sequence consisting of 0 and 1 . Each set of $k(1 \leq k \leq 4)$ bits of the watermark was embedded in an $8 \times 8$-pixel block of $Y$ component. We used PSNR for the evaluation of image quality. PSNR was calculated for 


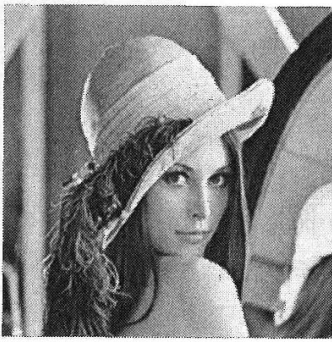

(a) Lena

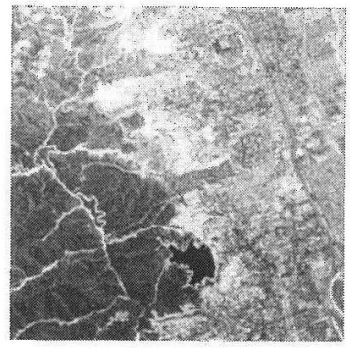

(c) Aerial

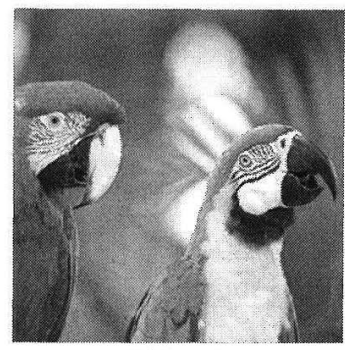

(e) Parrots

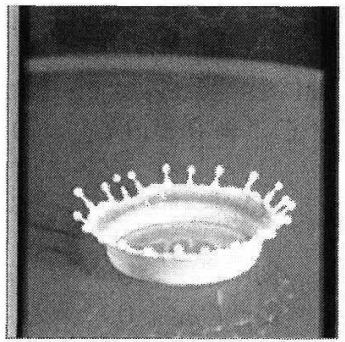

(g) Milkdrop

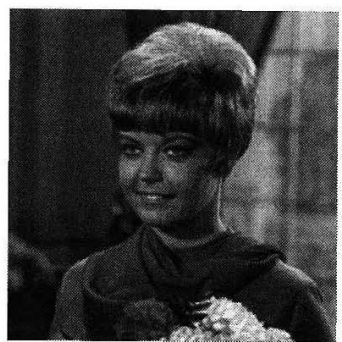

(b) Girl

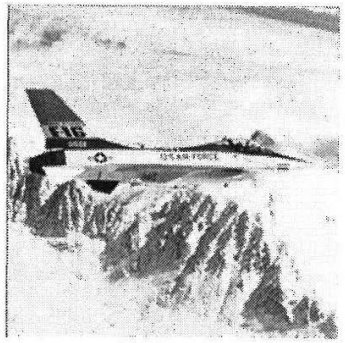

(d) Airplane

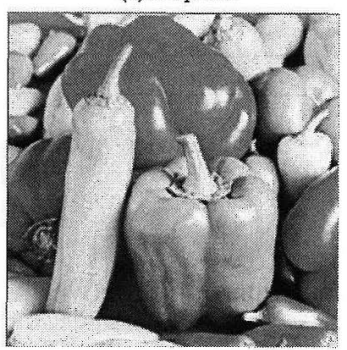

(f) Pepper

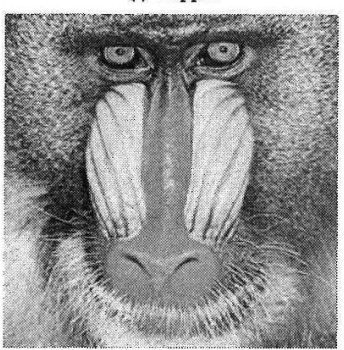

(h) Mandrill
Fig. 4 Original images.

an original JPEG image and the watermarked JPEG image which were converted to BMP format.

\subsection{The Number of Embeddable Blocks}

In the proposed method, the symbol of a watermark cannot be embedded in the $8 \times 8$-pixel block where $C_{63} \neq 0$. Then we investigated the relation between the number of embeddable blocks and JPEG quality. Figure 5 shows the relation between the number of embeddable blocks and JPEG quality for each image. Now, the maximum number of blocks is 1024.

As shown in Fig. 5, the higher JPEG quality is, the less the number of embeddable blocks is. It is because the values of quantization table decrease and the number of the blocks where $C_{63}=0$ decreases as JPEG quality increases. "Mandrill" has the much smaller number of em-

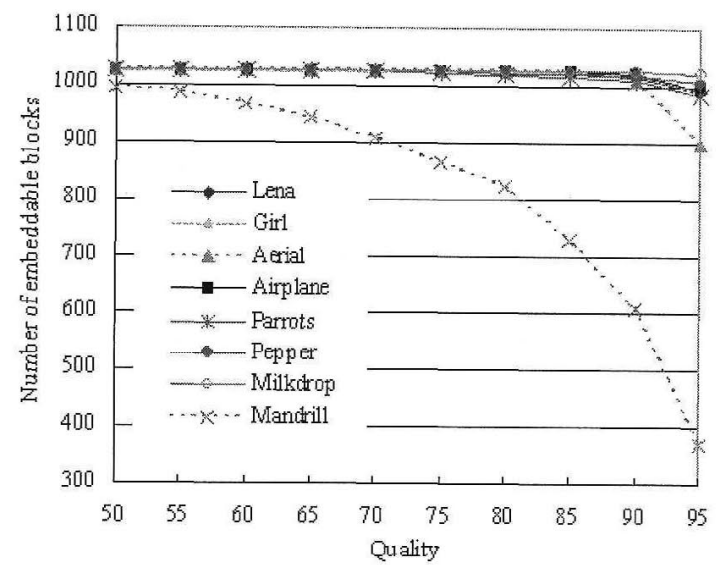

Fig. 5 The number of embeddable blocks.

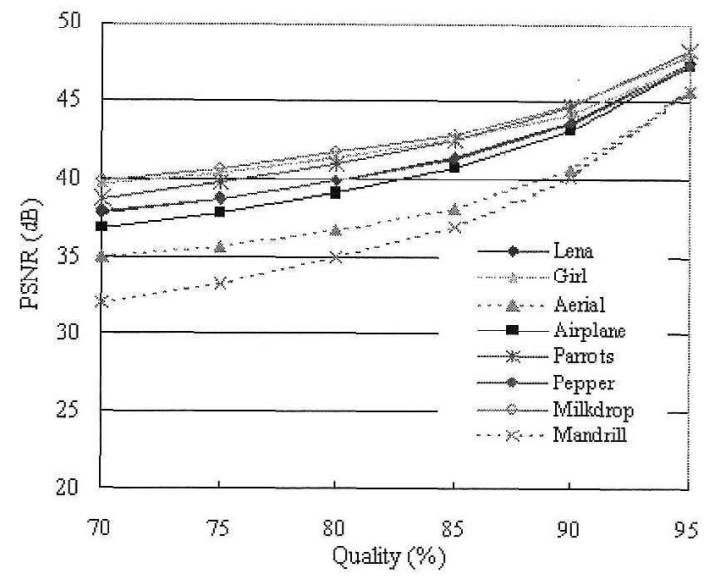

Fig. 6 Relation between JPEG quality and PSNR $(n=1)$.

beddable blocks than other images. It is because "Mandrill" contains more high-frequency components and so contains more blocks where $C_{63} \neq 0$.

The method in [6] embeds 154 bytes of payload in "Lena" image of $512 \times 512$ pixels ${ }^{\dagger}$, while the proposed method can embed up to 512 bytes of payload in "Lena" image of $512 \times 512$ pixels when $n=1$. On the other hand, the maximum payload of the method in [7] is greater than that of the proposed method. However, the performance, which is the relation between payload and PSNR, of the proposed method of $k \leq 3$ is almost the same as that of the method in [7] though the images used in the experiment are different.

\subsection{Image Quality}

Figure 6 shows the relation between PSNR and JPEG quality when $n=1$. Figure 7 shows the relation between PSNR and $k$ when JPEG quality is $90 \%$. Now, $n=2^{k-1}$.

As shown in Fig. 6, the higher JPEG quality is, the higher PSNR is. The symbols of a watermark are embedded in high-frequency DCT coefficients when JPEG quality is

\footnotetext{
${ }^{\dagger}$ It is written in [6] that the payload is 128 bytes, but it was corrected to 154 bytes later.
} 
Table 1 Increase of file-size (bytes) $(n=1)$.

\begin{tabular}{c|c|r|r|r|r|r|r}
\hline Image & size & $70 \%$ & $75 \%$ & $80 \%$ & $85 \%$ & $90 \%$ & $95 \%$ \\
\hline \multirow{3}{*}{ Lena } & watermarked & 11663 & 12784 & 14478 & 16846 & 21012 & 30016 \\
& original & 11371 & 12489 & 14198 & 16564 & 20722 & 29758 \\
& increase & 292 & 295 & 280 & 282 & 290 & 258 \\
\hline \multirow{3}{*}{ Girl } & watermarked & 9408 & 10339 & 11847 & 14084 & 17942 & 26996 \\
& original & 9130 & 10056 & 11567 & 13787 & 17652 & 26689 \\
& increase & 278 & 283 & 280 & 297 & 290 & 307 \\
\hline \multirow{3}{*}{ Aerial } & watermarked & 16527 & 18271 & 20946 & 24746 & 31309 & 45232 \\
& original & 16240 & 17991 & 20664 & 24451 & 31028 & 45028 \\
& increase & 287 & 280 & 282 & 295 & 281 & 204 \\
\hline \multirow{3}{*}{ Airplane } & watermarked & 12389 & 13480 & 15189 & 17776 & 22125 & 31450 \\
& original & 12085 & 13210 & 14900 & 17488 & 21835 & 31166 \\
& increase & 304 & 270 & 289 & 288 & 290 & 284 \\
\hline \multirow{3}{*}{ Parrots } & watermarked & 10075 & 10980 & 12428 & 14437 & 18058 & 25795 \\
& original & 9790 & 10700 & 12140 & 14147 & 17770 & 25510 \\
& increase & 285 & 280 & 288 & 290 & 288 & 285 \\
\hline & watermarked & 13150 & 14327 & 16221 & 18953 & 23689 & 33978 \\
& original & 12865 & 14038 & 15936 & 18671 & 23408 & 33719 \\
& increase & 285 & 289 & 285 & 282 & 281 & 259 \\
\hline \multirow{3}{*}{ Milkdrop } & watermarked & 9798 & 10770 & 12225 & 14297 & 17742 & 25827 \\
& original & 9512 & 10462 & 11943 & 13990 & 17477 & 25555 \\
& increase & 286 & 308 & 282 & 307 & 265 & 272 \\
\hline
\end{tabular}

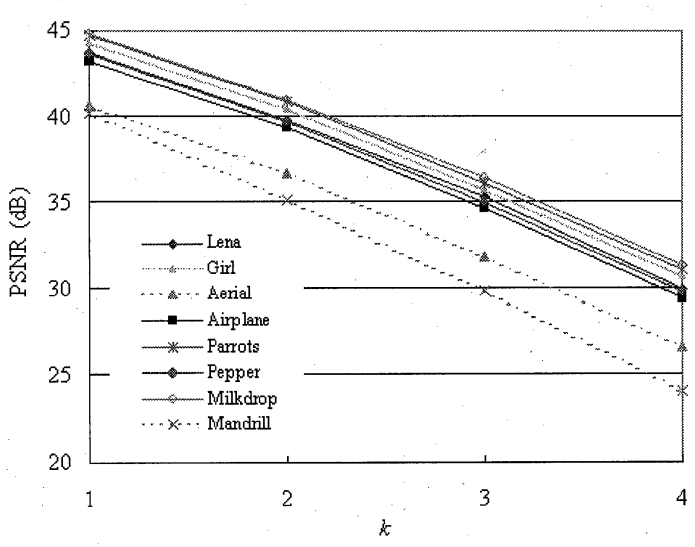

Fig. 7 Relation between $k$ and PSNR (Quality 90\%).

high. So it is because the influence of modification of highfrequency DCT coefficients on PSNR is less that PSNR is higher when JPEG quality is higher.

As shown in Fig. 7, PSNR (dB) almost linearly decreases as $k$ increases. The same tendency was shown for other JPEG qualities.

\subsection{Increase of File-Size by Watermark Embedding}

Table 1 shows the watermarked file-size (bytes), the original file-size (bytes) and the increase of file-size (bytes) by watermark embedding for each JPEG quality. As shown in Table 1, the increase of file-size by embedding a watermark is almost constant in spite of JPEG quality. In other words, the higher JPEG quality is, the less the rate of increase of file-size is, where the rate of increase of file-size means the ratio of the increase of file-size by embedding a watermark to the size of an original JPEG image. It is because the size of an original JPEG image is larger for higher JPEG quality that the rate of increase of file-size decreases.

\section{Conclusion}

We have proposed a reversible watermarking method for JPEG images. The proposed method embeds a watermark into a JPEG image, and it extracts the watermark from the watermarked JPEG image and at the same time can recover the watermarked JPEG image to an unwatermarked JPEG image. Moreover, the proposed method is easy to implement. We have investigated the number of embeddable blocks, the quality of watermarked images, and the increase of file-size by embedding a watermark.

\section{References}

[1] K. Matsui, Fundamentals of digital watermarking, Morikita Shuppan, 1998.

[2] J. Tian, "Reversible data embedding using a difference expansion," IEEE Trans. Circuits Syst. Video Technol., vol.13, no.8, pp.890-896, 2003.

[3] A.M. Alattar, "Reversible watermark using the difference expansion of a generalized integer transform," IEEE Trans. Image Process., vol.13, no.8, pp.1147-1156, 2004.

[4] M.U. Celik, G. Sharma, A.M. Tekalp, and E. Saber, "Lossless generalized-LSB data embedding," IEEE Trans. Image Process., vol.14, no.2, pp.253-265, 2005.

[5] L. Kamstra and H.J.A.M. Heijmans, "Reversible data embedding into images using wavelet techniques and sorting," IEEE Trans. Image Process., vol.14, no.12, pp.2082-2090, 2005.

[6] H. Sakai, M. Kuribayashi, and M. Morii, "Reversible data hiding technique for JPEG algorithm," Proc. SITA2007, pp.571-576; 2007.

[7] G. Xuan, Y.Q. Shi, Z. Ni, P. Chai, X. Cui, and X. Tong, "Reversible data hiding for JPEG images based on histogram pairs," 4th Inter. Conf., ICIAR 2007, LNCS 4633, pp.715-727, Springer-Verlag, 2007. 